\title{
Mindfulness-Based Intervention in School Psychology
}

\author{
Tyler L. Renshaw ${ }^{1} \cdot$ Aaron J. Fischer $^{2} \cdot$ David A. Klingbeil $^{3}$
}

Published online: 13 November 2017

(C) California Association of School Psychologists 2017

Interest in mindfulness and mindfulness-based intervention (MBI) continues to grow in both psychology and education. Although there is some dispute over the defining characteristics of mindfulness (Van Dam et al. 2017), there is general consensus that the construct consists of at least two interrelated phenomena: orienting one's attention to the here-and-now, while responding to the contents of one's awareness (e.g., thoughts and feelings) in a welcoming and accepting way (Bishop et al. 2004). MBI, then, can be understood as any technique or procedure that activates mindfulness - or trains mindfulness skills - for the purpose of bringing about a therapeutic outcome (Renshaw 2016). Within the past couple years, special journal issues devoted to the use of mindfulness and MBI in schools have been published in Mindfulness (Felver and Jennings 2016) and Psychology in the Schools (Renshaw and Cook 2017). The intent of this special issue is to further the scholarly discourse on this topic by highlighting applications of MBI that are particularly relevant to school psychologists. Prior to introducing the articles included in this issue, we provide some brief but necessary groundwork, to help contextualize the studies that follow. We begin by overviewing the state-of-the-science regarding MBI with youth and in schools, and then move to describe the potential role of MBI in school psychology.

Tyler L. Renshaw trenshaw@1su.edu

1 Department of Psychology, Louisiana State University, 236 Audubon Hall, Baton Rouge, LA 70803, USA

2 Department of Educational Psychology, University of Utah, Salt Lake City, UT, USA

3 Department of Educational Psychology, University of WisconsinMilwaukee, Milwaukee, WI, USA

\section{The State-of-the-Science of MBI}

A few meta-analyses have been conducted in recent years for the purpose of synthesizing and quantifying the available empirical evidence regarding the use of MBI with youth and in schools. The first published meta-analysis was conducted by Zenner et al. (2014), who focused specifically on studies testing MBI in school settings. They identified 24 group-design studies completed through August, 2012, including several unpublished studies $(k=8)$. Findings from this analysis indicated MBI had a small positive effect across outcomes for both pre-post comparisons $(g=0.41)$ and controlled comparisons $(g=0.40)$. Follow-up analyses indicated some differential effects across outcome domains, with moderate effects observed on measures of cognitive performance (pre-post $g=0.68$; controlled $g=0.80$ ) and small effects on measures of stress (pre-post $g=0.36$; controlled $g=0.39$ ) and emotional problems (pre-post $g=0.38$; controlled $g=0.36$ ).

Another meta-analysis, conducted by Zoogman et al. (2015), identified 20 group-design studies targeting MBI with youth in school and other community settings, published between 2004 and 2011. Results from their analysis were similar to those of Zenner et al. (2014), showing that MBI had a small therapeutic effect across all youth outcomes $(\mathrm{del}=0.23)$. Follow-up, exploratory analyses indicated that MBI had consistently small positive effects across three more targeted outcome domains: mindfulness ( $d e l=0.28)$, psychological symptoms $(\mathrm{del}=0.37)$, and non-symptom-oriented outcomes (del$=0.21$ ). A similar meta-analysis, albeit with different inclusion criteria, was carried out by Kallapiran and colleagues (2015), who identified 11 studies conducted with youth across settings, published through January, 2014. This study differed from Zoogman et al. (2015) given that inclusion criteria were limited to randomized controlled trials targeting mental health outcomes (i.e., depression, anxiety, and stress). Findings from 
this latter analysis were similarly favorable, indicating smallto-large therapeutic effects for each mental health outcome domain (Kallapiran et al. 2015).

A more recent and more comprehensive meta-analysis of group-design studies testing MBI with youth was conducted by Klingbeil et al. (2017b). This analysis included 76 studies, carried out in both school and non-school settings, published prior to January, 2016. Results indicated MBI had a small overall therapeutic effect for both pre-post comparisons $(g=0.31)$ and controlled comparisons $(g=0.32)$. Findings from 12 controlled studies that included follow-up assessments indicated that these positive effects were maintained over time $(g=0.40)$. Additional analyses showed consistently positive small-to-moderate effects across a variety of therapeutic processes and target outcomes, including mindfulness $(g=0.51)$, meta-cognition and cognitive flexibility $(g=0.40)$, attention $(g=0.29)$, emotional and behavioral regulation ( $g=0.32)$, academic achievement and school functioning ( $g=0.39)$, externalizing problems $(g=0.30)$, internalizing problems $(g=0.39)$, negative emotion and subjective distress $(g=0.25)$, positive emotion and self-appraisal $(g=0.28)$, physical health $(g=0.28)$, and social competence and prosocial behavior $(g=0.37)$. Interestingly, this analysis also reported that the setting in which MBI was implemented with youth (classified as either "school" or "other") did not moderate intervention effectiveness (Klingbeil et al. 2017b).

To date, the only meta-analysis to take up single-case design studies - as opposed to group-design research - is that conducted by Klingbeil et al. (2017a). Their analysis included 10 studies published between 2006 and 2014, all of which targeted youth exhibiting disruptive behavior. Results from their analysis indicated that MBI had a moderate effect on reducing disruptive behavior during treatment phases $(g=1.04$, TauU $=0.59)$ and that these positive effects were retained during maintenance phases $(g=1.41$, TauU $=0.71)$. Beyond using a different approach to research design, the studies included in this meta-analysis are remarkable compared to the group-design research synthesized in the other meta-analyses because the majority (a) consisted of youth with psychiatric diagnoses (e.g., attention-deficit/hyperactivity disorder and autism spectrum disorder), (b) used interventions that were structured primarily as direct skills training (as opposed to treatment packages consisting of both psychoeducation and skills training), and (c) used dependent measures of overt, observable behavior.

Taken together, findings from these five meta-analyses allow for a few tentative conclusions regarding the current stateof-the-science of MBI with youth and in schools. First, and probably most importantly, MBI appears to be safe and unlikely to yield iatrogenic effects with youth. Second, MBI appears to be generally beneficial, having at least small therapeutic effects across a variety of valued outcome domainsincluding school performance, cognitive skills, mental health, and physiological well-being. Finally, MBI seems to be useful in multiple service delivery contexts, having proved effective with both general and clinical populations of youth, in both school and other community settings.

Given the substantial number of studies conducted and the consistent positive results found across meta-analyses, we suggest that MBI can be considered an empirically supported approach to intervention. Yet, there are two cautionary points that should temper enthusiasm for this conclusion. One key point is that educational and psychological research have produced other evidence-based interventions that appear to be more effective for targeting the same outcome domains (Klingbeil et al. 2017b) - and so, until empirical research comparing the differential effectiveness of mindfulness with other approaches is conducted, there is no scientific warrant for preferring MBI over other well-validated approaches for improving academic skill proficiency (e.g., direct instruction of academic skills themselves) or reducing disruptive behavior in the classroom (e.g., group contingencies targeting problem behavior), for example. Another important point is that although MBI appears to work to change a variety of behaviors for the better, we do not yet have a scientific understanding of why or how they actually do so. For example, we have no empirical guidance regarding how intervention dosage may moderate effectiveness (Felver and Jennings 2016), or which components within MBI treatment packages are necessary versus superfluous (Renshaw and Cook 2017), or how much training and experience with mindfulness is required to effectively implement MBI (Klingbeil et al. 2017b). There is also little evidence supporting the behavior-change mechanisms that are purported to drive MBI (Klingbeil et al. 2017a). Thus, although the state-of-the-science so far is promising, it is also far from complete. Much more research is needed to both understand the workings of MBI and, relatedly, optimize the composition of MBI for use with youth and in schools.

\section{The Role of MBI in School Psychology}

Considering the promising evidence reviewed above, some scholars suggest that MBI may be a useful intervention approach for school psychologists to integrate within multitiered systems of support (Renshaw et al. 2015; Felver et al. 2013). At the universal level (Tier 1), MBI could be used for problem prevention and wellness promotion purposes and would consist of implementing a mindfulness-training curriculum within school-wide or class-wide settings. The majority of MBI developed so far have been packaged and marketed for this particular level, and the majority of studies investigating MBI with youth have been conducted at this level as well (Klingbeil et al. 2017b). Examples and descriptions of available curricula that school psychologists might consider-such as Mindful Schools and Inner Explorer - can be found in the 
review article of Semple et al. (2017). MBI curricula are also available to target the well-being of teachers and other school staff (e.g., Cultivating Awareness and Resilience in Education; Jennings et al. 2013); yet, there have been fewer empirical investigations of these programs than of those targeting youth directly.

Although universal-focused MBIs are all primarily oriented toward training mindfulness, the majority also include adjunct components, such as psychoeducation and other skills training that targets emotional and behavioral regulation. Additionally, mindfulness training itself can be included as an adjunct or minor component within other universal-level interventions, such as social-emotional learning (SEL) curricula for youth (e.g., Strong Kids; Gueldner and Feuerborn 2016) or general wellness promotion programs for teachers (e.g., ACHIEVER Resilience Curriculum; Cook et al. 2017). Despite the fact that there is little evidence to guide the selection or preference of MBI curricula compared to other universal-level interventions, we suggest that school psychologists can have confidence that universal-level MBIs are likely to be similarly effective to other approaches for reducing problems and promoting wellness that are commonly used at this level, such as SEL programming (cf. Durlak et al. 2011) and traditional mental health prevention programming (cf. Durlak and Wells 1997).

At the targeted level (Tier 2), MBI have been recommended for intervening with at-risk students within the context of small-group service delivery, conducted outside of the classroom yet still within the bounds of the regular school day. The curricula used to guide MBI at this level could be similar to those used at the universal level (see Semple et al. 2017 and Gueldner and Feuerborn 2016), yet would be implemented with greater intensity. These interventions could also be youth adaptations of more traditional, group-oriented mindfulnessbased treatment protocols developed in clinical settings, such as Mindfulness-Based Stress Reduction or Mindfulness-Based Cognitive Therapy (Felver et al. 2013). The important thing is that students receiving MBI at the targeted level should be grouped according to similar kinds of problem behavior (e.g., internalizing or externalizing), and that the MBI itself is geared toward helping youth learn mindfulness skills to address their specific presenting problems. Although MBIs have been used effectively with teachers and school staff at the universal level (e.g., Jennings et al. 2013), there is currently no available evidence suggesting they might be used for similar purposes at the targeted level.

Given the importance of progress monitoring in determining intervention effectiveness, school psychologists using MBI at the targeted level should select measures that gauge both the primary outcomes of interest to the group (e.g., internalizing or externalizing behaviors) and the therapeutic process outcomes specific to MBI (i.e., attention to the here-andnow and acceptance of the contents of one's awareness). Only by monitoring both types of outcomes will practitioners be able to infer that changes in student behavior (or the lack thereof) are likely a function (or not) of the acquisition of mindfulness skills. Reviews of available measures for assessing youths' mindfulness can be found in Eklund et al. (2017) and Goodman et al. (2017). Initial efforts are also underway to develop mindfulness measures that are specific to the school context (e.g., Frank et al. 2016; Renshaw 2017), but further studies supporting the use of these instruments are necessary before they can be considered empirically validated for progress monitoring purposes.

Finally, at the intensive level (Tier 3), MBI could be used within an individualized intervention plan to target a student's specific emotional or behavioral problems. Given that the majority of MBI curricula developed for youth are intended for use within large-group or small-group settings, and that the majority of research supporting these interventions has been conducted at the group level, there is far less empirical support for using MBI at this particular level of service delivery. Yet, there are some curricula that are intended for use with individuals as well (e.g., Mindfulness-Based Cognitive Therapy for Anxious Children; Semple and Lee 2011), and there is evidence suggesting that individualized psychotherapeutic approaches that integrate MBI (e.g., acceptance and commitment therapy; Halliburton and Cooper 2015) can also be effective with youth. There is also a growing body of evidence indicating that the Soles of the Feet intervention, which is a manualized MBI that has so far only been tested with individuals, can effectively reduce student's disruptive behavior (Klingbeil et al. 2017a). Furthermore, considering the strong evidence indicating that mindfulness-based treatments are effective for adults with clinical-level depression and anxiety (Khoury et al. 2013), it seems plausible that MBI may eventually prove useful as an intensive-level approach for addressing students' emotional problems. But, direct research in schools - especially studies comparing the differential effectiveness of MBI with other empirically validated treatmentsis needed to actually prove this point. Thus, until research demonstrates otherwise, we suggest school psychologists use MBI at the intensive level only as an adjunct intervention component, not as the primary approach to treatment.

\section{This Special Issue}

Given the context sketched above, we suggest the role of MBI within multitiered school psychological services is promising, yet it is clear further research is needed to guide the selection and implementation of MBI across all levels. This special issue contributes to the scholarly discourse toward this end by presenting applications of MBI that are relevant to the practice of school psychology at each of these three levels of service delivery. The first two articles, by Bannirchelvam et al. 
(2017) and Kielty et al. (2017), report on universal-level evaluations of MBI with elementary classrooms. Although there is already an extensive research base of outcome studies for MBI at this level, these studies contribute to the existing literature by offering qualitative investigations of the social validity of MBI-with findings suggesting that both students and teachers had favorable perceptions of the content and exercises included in mindfulness-training curricula. The next set of articles provides innovative examples of using MBI at the targeted level of service delivery. Specifically, Idler et al. (2017) tested the utility of MBI as an adjunct component within a standard reading fluency intervention for elementary students, whereas Milligan et al. (2017) explored the effects of a mindfulness-based mixed martial arts intervention with atrisk secondary students. Both of these studies provide useful starting points for others interested in similar innovative applications of MBI with target populations in schools.

The final set of articles provides examples of MBI used at the intensive level of service delivery. Rush et al. (2017) report on the effects of a mindfulness and biofeedback intervention carried out within a special education classroom consisting of students identified as "emotionally disturbed"-resulting in meaningful decreases in off-task behavior. Moreover, Felver et al. (2017) replicated and extended research on the Soles of the Feet intervention, targeting special education students classified as either "other health impaired" or "emotional/behavioral disability." Results from their study showed improved levels of academically engaged behavior for all students post-intervention. These contributions provide more evidence in favor of potentially using MBI as individualized interventions for students experiencing more severe behavior problems. In addition to these several original empirical studies, the last article included in this special issue is a conceptual piece by Alahari (2017), who argues that in addition to being useful for improving the outcomes of students and caregivers, MBI may also prove useful for targeting the social-emotional competencies and well-being of school psychologists themselves. Although this possibility has yet to be probed empirically, the argument appears sound and could be used as a warrant for initiating research toward this end.

In conclusion, we hope the articles in this special issue help advance the state-of-the-science and inform professional considerations regarding the use of MBI in school psychology. We express our appreciation to the authors who contributed to this issue and encourage those interested in their research to contact them directly. That said, we recognize that the scholarship presented herein does not resolve any of the critical concerns raised in our review above-such as the lack of dosage or component analyses, or the need for direct research investigating the differential effectiveness of MBI compared to other well-validated treatments - and so, we encourage those interested in progressing this line of work in school psychology to take up these points in future studies.
Although addressing these concerns empirically is likely to prove more challenging than simply testing the effects of MBI curricula or treatment packages, we suggest that these more complex lines of research are absolutely necessary in order to establish a true science-based understanding of how to best use MBI with youth and in schools.

\section{References}

Alahari, U. (2017). Supporting socio-emotional competence and psychological well-being through mindfulness practice. Contemporary School Psychology. https://doi.org/10.1007/s40688-017-0154-x.

Bannirchelvam, B., Bell, K. L., \& Costello, S. (2017). A qualitative exploration of primary school students' experience and utilisation of mindfulness. Contemporary School Psychology. https://doi.org/ 10.1007/s40688-017-0141-2.

Bishop, S. R., Lau, M., Shapiro, S., Carlson, L., Anderson, N. D., Carmody, J., et al. (2004). Mindfulness: a proposed operational definition. Clinical Psychology: Science and Practice, 11, 230-241.

Cook, C. R., Miller, F. G., Fiat, A., Renshaw, T. L., Frye, M., Joseph, G., $\&$ Decano, P. (2017). Promoting secondary teachers' well-being and intentions to implement evidence-based practices: randomized evaluation of the Achiever Resilience Curriculum. Psychology in the Schools, 54, 13-28. https://doi.org/10.1002/pits.21980.

Durlak, J. A., \& Wells, A. M. (1997). Primary prevention mental health programs for children and adolescents: a meta-analytic review. American Journal of Community Psychology, 25, 115-152. https:// doi.org/10.1023/A:1024654026646.

Durlak, J. A., Weissberg, R. P., Dymnicki, A. B., Taylor, R. D., \& Schellinger, K. B. (2011). The impact of enhancing students' social and emotional learning: a meta-analysis of school-based universal interventions. Child Development, 82, 405-432. https://doi.org/10. 1111/j.1467-8624.2010.01564.x.

Eklund, K., O’Malley, M., \& Meyer, L. (2017). Gauging mindfulness in children and youth: school-based applications. Psychology in the Schools, 54, 101-114. https://doi.org/10.1002/pits.21983.

Felver, J. C., \& Jennings, P. A. (2016). Applications of mindfulness-based interventions in school settings: an introduction. Mindfulness, 7, 14. https://doi.org/10.1007/s12671-015-0478-4.

Felver, J. C., Doerner, E., Jones, J., Kaye, N. C., \& Merrell, K. W. (2013). Mindfulness in school psychology: applications for intervention and professional practice. Psychology in the Schools, 50, 531-547. https://doi.org/10.1002/pits.21695.

Felver, J. C., Felver, S. L., Margolis, K. L., Ravitch, N. K., Romer, N., \& Horner, R. H. (2017). Effectiveness and social validity of the Soles of the Feet mindfulness-based intervention with special education students. Contemporary School Psychology. https://doi.org/10. 1007/s40688-017-0133-2.

Frank, J. L., Jennings, P. A., \& Greenberg, M. T. (2016). Validation of the Mindfulness in Teaching Scale. Mindfulness, 7, 155-163. https:// doi.org/10.1007/s12671-015-0461-0.

Goodman, M. S., Madni, L. A., \& Semple, R. J. (2017). Measuring mindfulness in youth: review of current assessments, challenges, and future directions. Mindfulness. Advance online publication. https://doi.org/10.1007/s12671-017-0719-9.

Gueldner, B. A., \& Feuerborn, L. L. (2016). Integrating mindfulnessbased practices into social and emotional learning: a case application. Mindfulness, 7, 164-175. https://doi.org/10.1007/s12671-0150423-6.

Halliburton, A. E., \& Cooper, L. D. (2015). Applications and adaptations of acceptance and commitment therapy (ACT) for adolescents. 
Journal of Contextual Behavioral Science, 4, 1-11. https://doi.org/ 10.1016/j.jcbs.2015.01.002.

Idler, A. M., Mercer, S. H., Starosta, L., \& Bartfai, J. M. (2017). Effects of a mindful breathing exercise during reading fluency intervention for students with attentional difficulties. Contemporary School Psychology. https://doi.org/10.1007/s40688-017-0132-3.

Jennings, P. A., Frank, J. L., Snowberg, K. E., Coccia, M. A., \& Greenberg, M. T. (2013). Improving classroom learning environments by Cultivating Awareness and Reslience in Education (CARE): Results of a randomized controlled trial. School Psychology Quarterly, 28, 375-390. https://doi.org/10.1037/ spq0000035

Kallapiran, K., Koo, S., Kirubakaran, R., \& Hancock, K. (2015). Effectiveness of mindfulness in improving mental health symptoms of children and adolescents: A meta-analysis. Child and Adolescent Mental Health, 20, 182-194. https://doi.org/10.1111/camh.12113

Khoury, B., Lecomte, T., Fortin, G., Masse, M., Therien, P., Bouchard, V., et al. (2013). Mindfulness-based therapy: a comprehensive metaanalysis. Clinical Psychology Review, 33, 763-771. https://doi.org/ 10.1016/j.cpr.2013.05.005.

Kielty, M., Gilligan, T., Staton, R., \& Curtis, N. (2017). Cultivating mindfulness with third grade students via classroom-based interventions. Contemporary School Psychology. https://doi.org/10.1007/ s40688-017-0149-7.

Klingbeil, D. A., Fischer, A. J., Renshaw, T. L., Bloomfield, B. S., Polakoff, B., Willenbrink, J. B., Copek, R. A., \& Chan, K. T. (2017a). Effects of mindfulness-based intervention on disruptive behavior: a meta-analysis of single-case research. Psychology in the Schools, 54, 70-87. https://doi.org/10.1002/pits.21982.

Klingbeil, D. A., Renshaw, T. L., Willenbrink, J. B., Copek, R. A., Chan, K. T., Haddock, A., Yassine, J., \& Clifton, J. (2017b). Mindfulnessbased interventions with youth: a comprehensive meta-analysis of group-design studies. Journal of School Psychology, 63, 77-103. https://doi.org/10.1016/j.jsp.2017.03.006.

Milligan, K., Cosme, R., Miscio, M. W., Mintz, L., Hamilton, L., Cox, M., Woon, S., Gage, M., \& Phillips, M. (2017). Integrating mindfulness into mixed martial arts training to enhance academic, social, and emotional outcomes for at-risk high school students: a qualitative exploration. Contemporary School Psychology. https://doi.org/ 10.1007/s40688-017-0142-1.

Renshaw, T. L. (2016). Using so-called mind-body practices to promote youths' wellbeing: reflections on therapeutic outcomes, strategies, and processes. International Journal of School \& Educational Psychology, 4, 116-121. https://doi.org/10.1080/21683603.2016. 1130587.
Renshaw, T. L. (2017). Preliminary development and validation of the Mindful Student Questionnaire. Assessment for Effective Intervention, 42, 168-175. https://doi.org/10.1177/ 1534508416678971

Renshaw, T. L., \& Cook, C. R. (2017). Mindfulness in the schoolshistorical roots, current trends, and future directions. Psychology in the Schools, 54, 5-12. https://doi.org/10.1002/pits.21978.

Renshaw, T. L., Bolognino, S. J., Fletcher, S. P., \& Long, A. C. J. (2015). Using mindfulness to improve well-being in schools. NASP Communiqué, 43(6), 4-8.

Rush, K. S., Golden, M. E., Mortenson, B. P., Albohn, D., \& Horger, M. (2017). The effects of a mindfulness and biofeedback program on the on- and off-task behaviors of students with emotional behavioral disorders. Contemporary School Psychology. https://doi.org/10. 1007/s40688-017-0140-3.

Semple, R. J., \& Lee, J. (2011). Mindfulness-based cognitive therapy for anxious children. Oakland: New Harbinger.

Semple, R. J., Droutman, V., \& Reid, B. A. (2017). Mindfulness goes to school: things learned (so far) from research and real-world experiences. Psychology in the Schools, 54, 29-52. https://doi.org/10. 1002/pits.21981.

Van Dam, N. T., van Vugt, M. K., Vago, D. R., Schmalzl, L., Saron, C. D., Olendzki, A., et al. (2017). Mind the hype: a critical evaluation and prescriptive agenda for research on mindfulness and meditation. Perspectives on Psychological Science. Advance online publication. https://doi.org/10.1177/1745691617709589.

Zenner, C., Herrnleben-Kurz, S., \& Walach, H. (2014). Mindfulnessbased interventions in schools: a systematic review and meta-analysis. Frontiers in Psychology, 5, 603-620. https://doi.org/10.3389/ fpsyg.2014.00603.

Zoogman, S., Goldberg, S. B., Hoyt, W. T., \& Miller, L. (2015). Mindfulness interventions with youth: a meta-analysis. Mindfulness, 6, 290-302. https://doi.org/10.1007/s12671-013$0260-4$.

Tyler L. Renshaw PhD is an Associate Professor in the Department of Psychology at Louisiana State University.

Aaron J. Fischer PhD is an Assistant Professor in the Department of Educational Psychology at the University of Utah.

David A. Klingbeil PhD is an Assistant Professor in the Department of Educational Psychology at the University of Wisconsin-Milwaukee. 